\title{
Comment
}

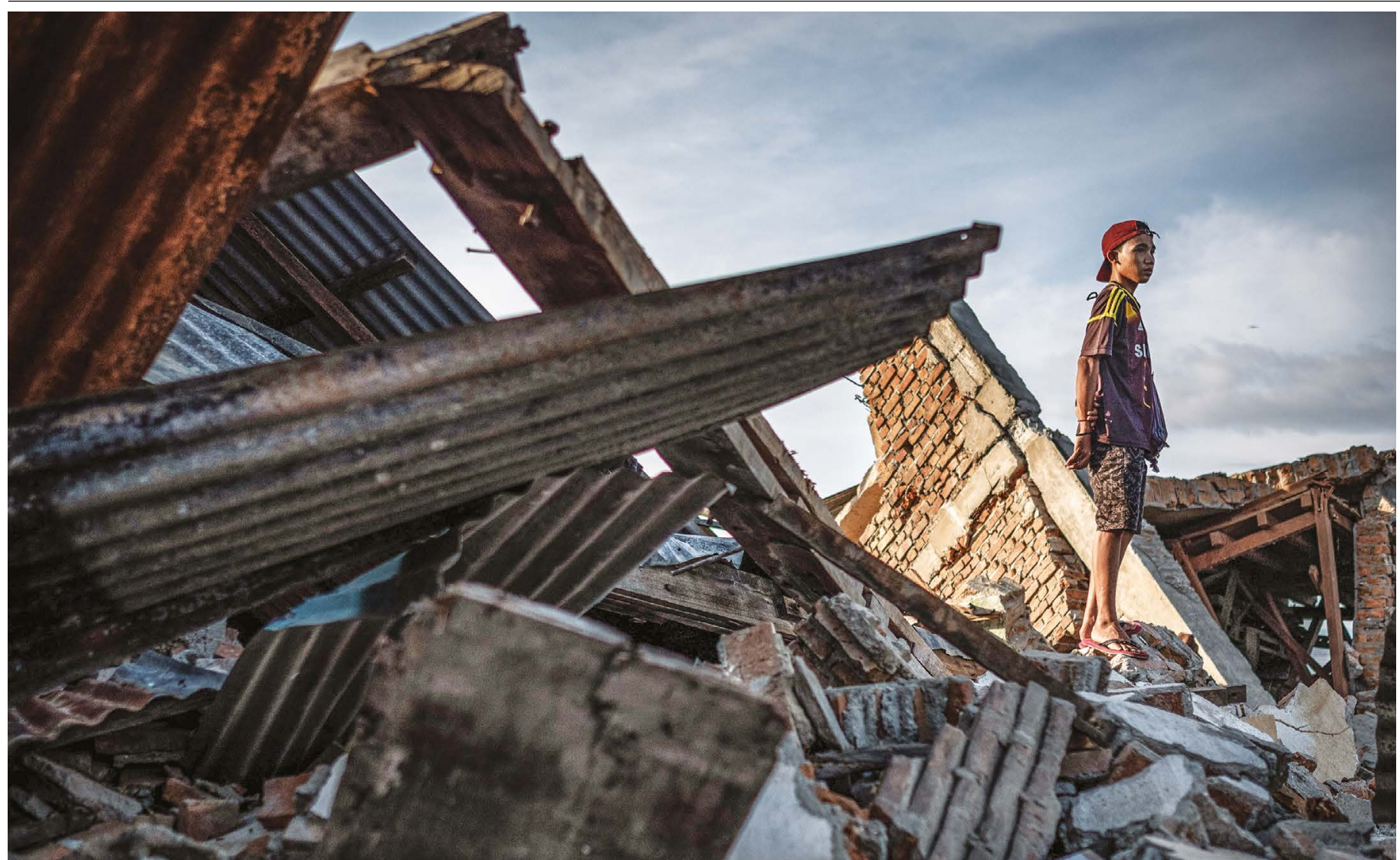

A collapsed building in the city of Palu in Sulawesi, Indonesia, after a magnitude-7.5 earthquake hit the region in September 2018.

\section{Disaster-zone research needs a code of conduct}

\author{
JC Gaillard \& Lori Peek
}

Study the effects of earthquakes, floods and other natural hazards with sensitivity to ethical dilemmas and power imbalances.

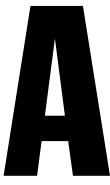

magnitude-7.0 earthquake rocked Anchorage, Alaska, in late November 2018. Roads buckled and chimneys tumbled from rooftops. Business operations were disrupted. Schools were damaged across the district. This was the largest earthquake to shake the region in a generation, and there was much to learn. What was the state of the infrastructure? Might further quakes occur? How did people respond? Teams of scientists and engineers from across the United States mobilized to conduct field reconnaissance in partnership with local researchers and practitioners. These efforts were coordinated through the clearing house set up by the Earthquake Engineering Research
Institute in Oakland, California, which provided daily in-person and online briefings, as well as a web portal for sharing data.

But researchers are not always so welcome in disaster zones. After the deadly Indian Ocean earthquake and tsunami on 26 December 2004, hundreds of academics from countries including Japan, Russia, France and the United States rushed to the region to collect perishable data. This influx of foreign scientists angered and fatigued some locals; many declined researchers' requests for interviews. The former governor of Aceh province, Indonesia, where more than 128,000 people died, described foreign researchers as "guerrillas applying hit-and-run tactics"1. 
Yet research on tsunami propagation and people's response to the event has led to improved warnings and emergency-response plans.

When, on 28 September 2018, an earthquake and tsunami hit the Indonesian island of Sulawesi, dozens of researchers found themselves unable to enter the country ${ }^{2}$. Indonesian law now requires foreign scientists to obtain a special visa before they can begin research. Data-collection protocols must be submitted to the government in advance and projects must have an Indonesian partner. Violators could face criminal charges and even prison.

This incident has inflamed a smouldering debate among disaster researchers. Some scholars argue that stringent administrative protocols violate researchers' rights and prevent the collection of crucial, potentially life-saving, data ${ }^{3}$. Others counter that such procedures protect survivors and preserve the integrity of local scientific efforts. For instance, concerns over studies placing undue burdens on overwhelmed groups - including grieving schoolchildren - led New Zealand to impose a moratorium on social-science research after the 2011 Christchurch earthquake ${ }^{4}$.

Here we argue that disaster research needs a culture shift. As in other branches of study involving human participants, ethical concerns should have the same primacy as research questions ${ }^{5}$. We call on the United Nations Office for Disaster Risk Reduction (UNDRR) to put forward a researcher-driven ethical code of conduct. This should advance disaster research, making it scientifically rigorous as well as locally and culturally grounded. After all, the UNDRR has a mandate "to ensure synergies among ... regional organizations and activities in socio-economic and humanitarian fields".

\section{Moral hazard}

Researchers working in disaster zones, with people whose culture might be different from their own, need to know how to interact with survivors as well as local officials and scholars, without adding to those people's problems.

There is no universal definition of ethical behaviour, and only a handful of countries have ethically informed guidelines for post-disaster research. In New Zealand, guiding principles from the Natural Hazards Research Platform advise that researchers must "avoid creating unnecessary anxiety by speculating to locals". The Philippines allows research on the trauma caused by disasters only in exceptional cases, such as when affected people want to share their feelings as a way to process the event. Brazil, like Indonesia, requires all researchers working in the country to have a special visa and an established local connection.

University ethics committees and national ethical review boards are unable to fill the gap. They tend to focus on studies in medicine and social sciences that involve human participants. They have little to say on how to investigate a collapsed building or a compromised coastal landscape. Yet studies by engineers or natural scientists have participants, too: local residents, scholars, guides and interpreters. Tsunami researchers might

\section{"Ethical concerns should have the same primacy as research questions."}

need to ask coastal dwellers about the height of waves; structural engineers assessing a collapsed stairwell might question the building's occupants about how they escaped.

\section{Towards a code of conduct}

Researchers equipped with an 'ethical toolkit' are better able to help affected populations ${ }^{6}$ without causing harm. Following the earthquake that struck Luzon island in the Philippines in April this year, research was coordinated by academics based in nearby Manila. They provided support deemed appropriate by those affected. A code of conduct could build on such successes and should consider the following three principles.

Have a clear purpose. Researchers should collectively identify knowledge gaps that future studies will fill. They should partner with affected people to establish emergent research priorities in dealing with a disaster. Such collaborative engagement can help to clarify where and when researchers will go into the field, what they will study, and who should be on the team. For example, psychologists and anthropologists might study and support local coping mechanisms; historians and civil engineers might collaborate to examine and promote resilient traditional architectural features when rebuilding homes in cyclone-affected areas.

The needs of local people should be central' ${ }^{7}$. Too often, research is driven by media coverage and politics. Disasters in heavily populated areas receive the most attention, but the cumulative impacts of smaller events can be just as devastating. For example, after the massive Nepal earthquake in April 2015, the impacts on infrastructure and the quality of shelters were widely studied, and aid donors gave millions of dollars to rebuild parts of Kathmandu. Yet in rural western Nepal, hundreds of villages cope with floods and landslides each year, unnoticed by the outside world.

A researcher code can help to redress the balance. For example, the Philippines requires that post-disaster projects demonstrate how they will meet the priorities of affected communities. New Zealand encourages researchers to defer collecting data unless the information will support responders. More relevant research could provide the evidence to inform and direct recovery funding to where needs really lie.

Respect local voices. Wealthy countries account for most disaster scholarship and funding. For example, more than $90 \%$ of articles published following Hurricane Katrina, which hit the southern United States in 2005, were by US researchers ${ }^{8}$. By contrast, fewer than $5 \%$ of publications on the 2010 Haiti earthquake were led by authors based in the country (see 'Unequal partners').

Similarly, $84 \%$ of articles published between 1977 and 2017 in Disasters, the flagship journal in the field, were led by authors based in countries of the Organisation for Economic Co-operation and Development (OECD). Yet 93\% of the people killed by large disasters over the same period lived in non-OECD countries, according to the EM-DAT disaster database?.

Outside researchers - who have not had their lives disrupted by disaster - are positioned to seek funding and might overlook local work and partners. After Hurricane Katrina in 2005, local experts in urban poverty, affordable housing and coastal land loss were passed over for grants ${ }^{10}$. And local and external priorities might differ. In 2011, following the Joplin tornado in Missouri, outside academics assessed damage to infrastructure. By contrast, locally based researchers were eager to learn how to support emotional health after witnessing a rise in post-traumatic stress in children and adults ${ }^{11}$. Both are important topics, but funding streams do not always follow local desires.

An understanding of local languages, policies and practices is essential and can improve response and speed recovery. After Katrina, 'culture brokers' helped survivors to make sense of government documents so that they could access aid quickly ${ }^{12}$. Nonetheless, much disaster research is still framed by narrow world views. Concepts such as vulnerability and resilience do not necessarily translate well $^{13}$. Even where equivalent terms exist, they might be felt to be irrelevant, because natural phenomena such as cyclones and floods are not always seen as hazards. In some religious 
traditions, volcanic eruptions are thought to reflect the emotions of deities, for instance. A lack of recognition of this nuance can affect the outcomes of risk-perception research as well as early-warning processes.

More discussions between disaster researchers inside and outside affected areas would shed light on these issues and could inform a more holistic research agenda. The Geotechnical Extreme Events Reconnaissance Association's ethics protocol might serve as a starting point. It encourages engineers to adhere to "high standards of professionalism" and to be "respectful of local customs, traditions, privacy, and rights of affected individuals" (see go.nature.com/32kptno). Government agencies, companies and non-governmental organizations should also be involved in such conversations, given that they are increasingly engaged in post-disaster data collection ${ }^{14}$.

Coordinate locals and outsiders. Projects that are uncoordinated can become irrelevant or redundant, and might overwhelm local people and responders. In 2013, survivors of Typhoon Yolanda (also known as Haiyan) in Tacloban in the Philippines were deluged with questionnaires, when their immediate concerns were to secure housing, food, clothing and education.

After Hurricane Harvey in the United States in 2017, officials at emergency operations centres struggled to decipher the credentials of dozens of researchers who descended on Houston, Texas, requesting access. Emergency managers also had to spend precious time revising researchers' survey questions to put them in a local context.

Foreign scientists sometimes approach local researchers to serve as translators or assistants. These locals have little power to direct the research strategy, even though their insights are valuable. They might feel unable to be critical even when they know the questions are wrong-headed. Even when they make substantial contributions, they might still be relegated to co-authorship - or no authorship - rather than being listed as the primary author.

Incoherent data and findings might confuse authorities and delay decisions. Volcanologists still argue about exactly when local communities should be evacuated. To help, the International Association of Volcanology and Chemistry of the Earth's Interior has produced

\section{"Much disaster research is still framed by narrow world views."}

guidelines on the roles and responsibilities of local and outside scientists, local authorities and the media.

Local researchers need to be identified quickly in a crisis. As a start, the Social Science Extreme Events Research (SSEER) network has produced a global map of social scientists who study hazards and disasters (see go.nature.com/2qfwezc). Regional SSEER councils ensure that those researchers remain involved after the event.

\section{First steps}

Discussions regarding a shared code of conduct could start through collaborative disaster-research initiatives that are under way worldwide. These have established strong coordinating structures and forums for information sharing, and include those in Latin America, Africa, the European Union and the Asia-Pacific region. They could also build on disaster-response initiatives from

\section{UNEQUAL PARTNERS}

Authorship of papers on disaster research can be dominated by researchers

outside the country affected, meaning that local expertise might be overlooked.

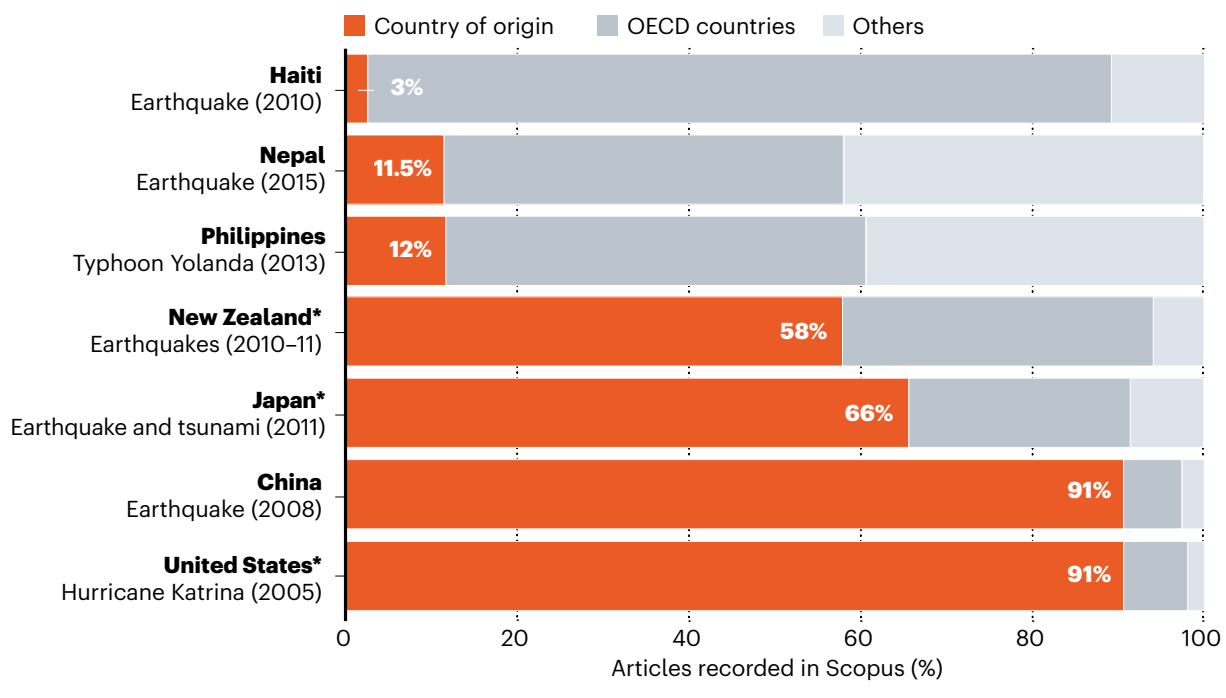

* 'OECD countries' category excludes the affected member country of the Organisation for Economic Co-operation and Development.

the medical sciences ${ }^{15,16}$.

The US National Science Foundation (NSF) now supports several extreme-events reconnaissance and research networks. These advance coordination and set scientific agendas in geotechnical and structural engineering, social sciences, near-shore systems, operations and systems engineering, and interdisciplinary science. The NSF-funded CONVERGE initiative (of which L.P. is the principal investigator) brings together leaders from these networks and major NSF facilities to support the development of guidance and data-sharing by hazards and disaster researchers. Other resources, including a set of free online training modules, are also available. These NSF initiatives are open to researchers globally, but they are led by researchers at US institutions.

Most countries do not provide ethical guidance for researchers, and universities have widely varying standards for the protection of study participants. The UNDRR is a trusted convener of scientists and practitioners globally. It could serve as a focal point for the development and implementation of an ethical code of conduct for researchers in disaster zones. As disasters unfold around the globe, the need for such a code of conduct becomes ever more urgent.

\section{The authors}

JC Gaillard is an associate professor in the School of Environment at the University of Auckland, New Zealand; and extraordinary professor in the Unit for Environmental Sciences and Management, North-West University, Potchefstroom, South Africa. Lori Peek is professor of sociology and director of the Natural Hazards Center, University of Colorado Boulder, USA; and principal investigator of the US National Science Foundation-supported CONVERGE, SSEER and ISEEER Initiatives. e-mails: jc.gaillard@auckland.ac.nz; lori.peek@colorado.edu

1. Missbach, A. Crit. Asian Stud. 43, 373-398 (2011). 2. Nature $562,317-318$ (2018).

3. Kendra, J. \& Gregory, S. in Disaster Research and the Second Environmental Crisis (eds Kendra, J., Knowles, S. G. \& Wachtendorf, T.) 319-341 (Springer, 2019).

4. Beaven, S., Wilson, T., Johnston, L., Johnston, D. \& Smith, R. Earthq. Spectra 32, 713-735 (2016).

5. Guillemin, M. \& Gillam, L. Qual. Inq. 10, 261-280 (2004).

6. Browne, K. E. \& Peek, L. Int. J. Mass Emerg. Disasters 32, 82-120 (2013).

7. Chambers, R. Rural Development: Putting the Last First (Longman, 1983).

8. Gaillard, J. C. Disasters 43, S7-S17 (2019).

9. Gaillard, J. C. \& Gomez, C. Jàmbá: J. Disaster Risk Stud. https://doi.org/10.4102/jamba.v7i1.120 (2015).

10. Barber, K. \& Haney, T. J. Sociol. Spectrum 36, 57-74 (2016).

11. Houston, J. B. et al. PLoS Curr. https://www.ncbi.nlm.nih. gov/pmc/articles/PMC4639320 (2015).

12. Browne, K. Standing in the Need (Univ. Texas Press, 2015).

13. Bankoff, G. Disasters 25, 19-35 (2001).

14. Miller, A. et al. Int. J. Environ. Res. Public Health 13, 676 (2016).

15. Sumathipala, A. et al. Asian Bioeth. Rev. 2, 124-142 (2007).

16. Collogan, L. K., Tuma, F., Dolan-Sewell, R., Borja, S. \& Fleischman, A. R. J. Trauma. Stress 17, 363-372 (2004). 